\title{
Modeling the Speed and Timing of American Sign Language to Generate Realistic Animations
}

\author{
Sedeeq Al-khazraji, Larwan Berke, Sushant Kafle, Peter Yeung, Matt Huenerfauth \\ Golisano College of Computing and Information Sciences \\ Rochester Institute of Technology (RIT), Rochester, NY, USA \\ sha6709@rit.edu, 1wb2627@rit.edu, sxk5664@rit.edu,pxy9548@rit.edu, matt.huenerfauth@rit.edu
}

\begin{abstract}
To enable more websites to provide content in the form of sign language, we investigate software to partially automate the synthesis of animations of American Sign Language (ASL), based on a human-authored message specification. We automatically select: where prosodic pauses should be inserted (based on the syntax or other features), the timeduration of these pauses, and the variations of the speed at which individual words are performed (e.g. slower at the end of phrases). Based on an analysis of a corpus of multisentence ASL recordings with motion-capture data, we trained machine-learning models, which were evaluated in a cross-validation study. The best model out-performed a prior state-of-the-art ASL timing model. In a study with native ASL signers evaluating animations generated from either our new model or from a simple baseline (uniform speed and no pauses), participants indicated a preference for speed and pausing in ASL animations from our model.
\end{abstract}

\section{Author Keywords}

American Sign Language, ASL, Animation, Prosodic Breaks, Pauses, Timing, Modeling.

\section{ACM Classification Keywords}

Human-centered computing $\rightarrow$ Accessibility $\rightarrow$ Empirical studies in accessibility. Computing methodologies $\rightarrow$ Artificial intelligence $\rightarrow$ Natural language processing $\rightarrow$ Natural language generation.

\section{BACKGROUND AND MOTIVATION}

There is great diversity among people who are Deaf or Hard of Hearing (DHH), across many demographic and lifeexperience characteristics. One aspect of this diversity is in regard to individuals' language and communication preferences, as well as their reading literacy skills in the written language used in their region of the world. In the U.S. context, some individuals who are DHH may prefer to communicate using American Sign Language (ASL) or feel

\footnotetext{
Permission to make digital or hard copies of all or part of this work for personal or classroom use is granted without fee provided that copies are not made or distributed for profit or commercial advantage and that copies bear this notice and the full citation on the first page. Copyrights for components of this work owned by others than ACM must be honored. Abstracting with credit is permitted. To copy otherwise, or republish, to post on servers or to redistribute to lists, requires prior specific permission and/or a fee. Request permissions from Permissions@acm.org.

ASSETS '18, October 22-24, 2018, Galway, Ireland

(C) 2018 Association for Computing Machinery. ACM ISBN 978-1-4503-5650-3/18/10 ..\$15.00 https://doi.org/10.1145/3234695.3236356
}

a strong personal association to the Deaf community. For instance, approximately 500,000 people consider ASL to be a primary form of communication [19]. Prior research, e.g. $[12,13]$, has discussed the language use and literacy characteristics of people who are Deaf, including:

- ASL is a distinct language from English, and it has a different word-order and linguistics, such that ASL is not simply a method of representing English sentences. ASL is a natural language, which uses hand shapes, movements, and orientation, as well as face/head/body movements to convey words and structure [2]. Furthermore, ASL has a unique historical development and distinct language community from English; thus, it is possible for an individual to be fluent in ASL, while having limited fluency in English.

- While there are many DHH individuals with excellent reading literacy skills and academic achievement, there are also some DHH individuals who have lower English literacy skills, due to diverse educational experiences or childhood language exposure. In fact, standardized testing in the U.S. has found that over a half of deaf adults graduating secondary school have an English reading literacy rate at the "fourth grade" level, which is the standard reading skill level for U.S. school children who are age 9 or 10 [28].

With this context, it is understandable that a barrier faced by many deaf adults in the U.S. is accessing information in the form of English text, including on websites [22]. While it is common for companies, organizations, or governments to offer versions of their website in multiple written languages, it is much less common to see websites that provide information content in the form of sign language. Researchers have argued that this is due to challenges faced in producing, managing, and updating video recordings of humans performing sign language [14]. As compared to the ease of updating a text file containing a written language, updating information in a video of sign language hiring a fluent signer and re-recording information content. An alternative solution is to provide computer animation of sign language on websites, but the time and resources needed for human animators to carefully produce fluent and understandable motions of a 3D virtual human character are also substantial [14]. Prior research presented at ASSETS, e.g. [13], and at other venues $[1,6,12,26]$ has investigated how to (partially) automate the creation of such animations, to reduce the time and skills needed by the human who is 


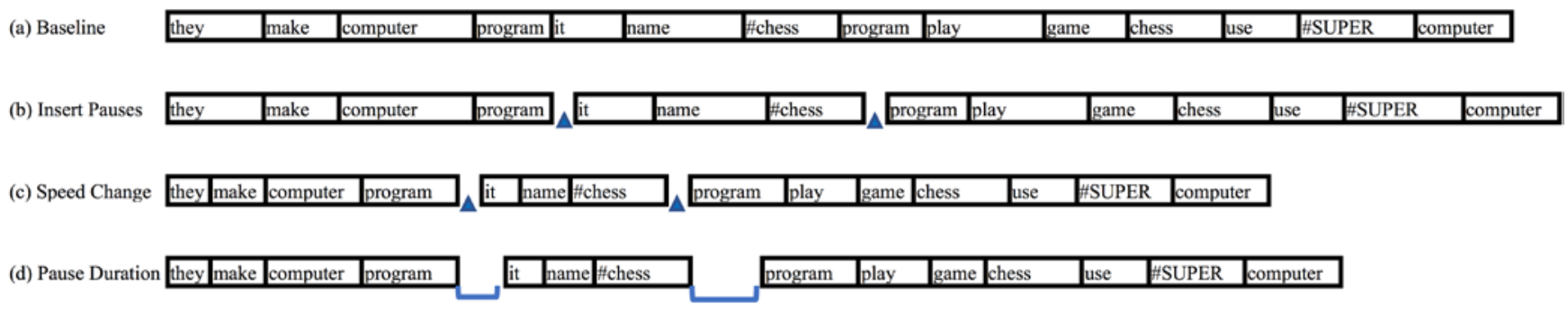

Figure 1: This series of timeline images show how an ASL animation system may sequence decisions regarding speed and timing: (a) The input to the system is a script, specifying the identity of the words to use in the sentence - drawn from an internal lexicon in the ASL animation system and using a 'default' fundamental rate of signing. (b) The system must select where during the word sequence pauses should be inserted. (c) Next, the system differentially adjusts the rate of signing for each individual word, based on a variety of linguistic factors. (d) Finally, the system selects the duration of the pauses during the animation. This model of ASL speed and timing is used throughout this paper, when discussing prior work and presenting our new ASL-Speed model.

authoring the message. Rather than a computer animator, a human who is skilled in ASL could author a symbolic representation of the message, which software could synthesize into a computer animation.

While the author of such an input script must be someone who is fluent in ASL (ideally a Deaf individual with native fluency in ASL who could translate the English text), we do not believe it is reasonable to burden the human author with making subtle numerical decisions about, e.g., the number of milliseconds to pause between words or the specific speed variations of individual words throughout a longer discourse. While fluent signers may be able to produce ASL (using their body) that contains these natural timing subtleties themselves, they may not be able to choose specific numerical values for all of these properties, for all words in a script for generating a computer animation.

\section{Goal of our Research}

Thus, the specific challenge we address in our research is, given a sequence of ASL words in a message (along with a limited amount of additional structural information provided by the human author, e.g. sentence boundaries), to automatically identify the following three speed and timing values for each individual word in the message:

- Is there a prosodic break (a pause) after this specific word? ASL signers will naturally pause at various locations during a message, typically more frequently at structural boundaries, e.g. as discussed in [24].

- If so, what is the time-duration of this break/pause? ASL signers are also more likely to use longer pauses at more important structural boundaries, e.g. [10].

- Given the overall signing rate that we seek to produce, what is the variation of this speed (slightly faster, slightly slower) for each particular word in the message? ASL signers will generally slow down at the end of sentences, or change their signing speed for individual words, for a variety of reasons [9, 29].

While these subtle timing values may seem negligible at first blush, prior perceptual studies of the quality of sign language animation (conducted with DHH participants) has revealed that minor variations in these three parameters can lead to significant differences in users' perception of the quality and understandability of animations [12]. Thus, we investigate how to automatically answer these questions, for each word in an input ASL script, by training machinelearning models on movement data from human ASL signers, from a pre-existing corpus of ASL containing motion-capture data and linguistic annotation. We have evaluated our models in two ways: (1) a model-based evaluation using cross-validation on this dataset (comparing the model to the current state-of-the-art model of speed and timing for ASL animations published at ASSETS'08 [12, 13]) and (2) a study with DHH participants who compared animations side-by-side (one based on this new model and the other a baseline timing model) and offered their subjective assessment of the animation quality and recommendations for future improvements.

\section{KEY CONCEPTS FOR ASL SPEED AND TIMING}

We began by examining prior linguistic research on speed and timing parameters of sign language. As shown in Figure 1, when conceptualizing sign-language speed and timing, is important to distinguish five components:

\section{\#1: Fundamental Rate (Words Per Second, Overall)}

Some signers, in some settings, are faster in their personal rate of signing than others. In a similar way, some speakers are "faster talkers." In this paper, we refer to this overall rate of signing communication as the signer's fundamental speed, and it can be expressed as the number of "words per second" that the signer tends to produce, as averaged over some longer span of language production. Prior linguistics researchers have analyzed videos of human signers to determine that typical fundamental rates of signing vary between 1.5 and 2.37 words per second, depending on context [2, 7, 8, 9]. In comparative studies, researchers found that ASL signing conveys information content at an equivalent rate as spoken English [2]. While ASL fundamental rate is lower (fewer words per second), ASL sentences tend to be shorter (consisting of fewer individual words per sentence) to convey the same ideas. 


\section{\#2: Base Duration (of a Word in a Lexicon)}

Each ASL sign consists of a particular number of movements of the hands, to particular locations in space or on the body. As such, ASL signs have a basic duration that varies from word to word, with some signs naturally taking longer to perform than others, since some ASL signs consist of a more complex or larger set of movements. Loosely, the reader may consider an analogy to spoken languages, in which spoken words vary in the number of syllabus they contain (e.g., "so" vs. "consequentially"). Some linguistics researchers have conducted work on lexicography of sign languages, in which they assemble lexicons (vocabulary lists or dictionaries) for various sign languages, often based on identifying large numbers of examples of a particular word used in multiple video recordings of sign language that have been analyzed, e.g. [20]. If enough examples can be identified for a particular word, researchers can estimate a typical range for the base duration of that word.

\section{\#3: Differential Signing Rate (of a Word as Performed)}

During a sentence or some longer span of signing, a signer will vary in the differential speeds at which each individual word is performed. For instance, as a signer approaches the end of a sentence, the final word(s) may be performed more slowly. Thus, the differential signing rate is a property of an individual word, as performed in an individual instance such that the sign may be performed more quickly or more slowly, for various contextual reasons. Notably, the specific amount of time that a signer uses to perform some word, on a particular occasion, is based on an interaction of all three of these factors above (fundamental rate, base duration, and differential rate). Prior linguistics researchers have investigated the speed of individual words in an ASL performance, by analyzing video recordings of human ASL signers $[9,29]$. There is a challenge in studying differential rate from recordings: When observing the amount of time someone requires to perform an ASL sign in a particular instance, the duration is based on the overall fundamental rate and the base duration of the word. Unless the researchers are able to calculate average speed for individual signers and average duration of a particular words, it is difficult to isolate the differential rate of a particular word in that performance.

\section{\#4: Pause Insertion}

Prior linguistics researchers have studied where ASL signers tend to pause, e.g. Grosjean et al. [10] based their analysis on observations of video recordings of native ASL signers. They conceptualized the decision as to whether a pause should occur at a particular word boundary as being binary in nature, i.e. they determined that at any word-toword boundary, there either exists or does not exist a pause at that location. (The reader may note that an alternative conceptualization would be to assume there is some amount of "pause" at every boundary - sometimes with duration 0 , meaning that there is no apparent pause.) In our work, we adopt the binary view of Grosjean at el. [10], as discussed in the "Methods" section below. During their analysis of videos, Grosjean et al. [10] determined that there were pauses at approximately $25 \%$ of word boundaries in an ASL passage. They also proposed a scheme for insertion of pauses based on the syntax structure of the sentence and proximity of a word boundary to other nearby pauses.

\section{\#5: Pause Duration}

In their analysis of videos of signing, Grosjean et al. [10] noted the duration of each pause they observed, and they calculated average pause duration, when the pause was at various key locations: 229 milliseconds between sentences, 134 milliseconds between conjoined sentences, 106 milliseconds between noun or verb phrases, 11 milliseconds if within verb phrases, etc. The main take-away from their analysis was that pause duration in ASL was related to the syntactic structure of the sentence. They proposed a scheme for predicting the duration of pauses at various syntactic locations [10], which was utilized in a prior ASL speed and timing model [12], which is the current state-of-the-art method for predicting speed and timing in ASL animation.

\section{SPEED AND TIMING IN SIGN LANGUAGE ANIMATION}

We have also considered prior research on modeling and predicting these parameters, to synthesize animations of sign language. There have been many research systems created with a goal of generating animations of sign language, e.g. see survey in [14]. In this literature survey, we discuss only a representative subset of prior sign language animation systems that have, in some way, addressed speed or timing factors. This section begins with a brief listing of the systems under discussion, followed by an analysis of how these systems may have addressed the five-component model of speed and timing outlined above:

- 2008 Model of Huenerfauth [12, 13]: The current state-of-the-art model for predicting these speed and timing parameters in ASL animation was presented by Huenerfauth at ASSETS'08 [13]. Because we often compare our current system to this model, we shall refer to this with the short name "2008 Model." This model consisted of several rules for calculating various timing parameters, but it required substantial (and time-consuming) input information, namely a full syntactic parse tree for every ASL sentence. This model utilized linguistic findings from $[9,10,11]$.

- Adamo-Villani and Wilbur [1]: This was a rule-based system for generating sign speed and pauses, to add prosodic elements into an animation of ASL being authored, based upon linguistic findings from [24, 29].

- eSign [16]: This project developed an animated signing avatar which could be used to convey information on European websites, e.g. government agencies; the system performed sequences of signs from a lexicon, based on a script designed by an author.

- Ebling and Glauert [6]: This was a system for translating announcements in a train station from a German text into Swiss German Sign Language.

- Segouat and Braffort [26]: This work was based on motion-capture data, as in our current study; here the authors re-combined elements of motion recordings to 
generate new animations and implemented some coarticulation models of how adjacent signs interact.

- Sign Smith Studio [30]: This was a commercially available product allowing users to create an animation of ASL, by assembling a sequence of words (from a lexicon provided) on a timeline and add other details.

Below, we discuss each of the five components of speed and timing, in regard to the systems listed above.

\section{Fundamental Rate in Animation Systems}

Sign Smith Studio [30] is a good example of an ASL animation scripting tool that enables a human user, who is knowledgeable of sign language, to create a message. This system did not have any automatic algorithms for predicting speed or timing factors, but it provided the human author of the message with a great degree of control, e.g. enabling users to adjust an individual speed multiplier for each word, modify the speed of the transitional movement between words, or add additional pause time (of any duration) after a word. This level of control came at the expense of effort from the author, who had to manually adjust all of these parameters, which could be difficult or time-consuming [30]. Notably, in regard to fundamental rate, the system provided the author with a "master speed control" slider that could be used to adjust the overall speed of the signer's movement.

Prior researchers have empirically investigated what fundamental rate of signing is preferred by $\mathrm{DHH}$ individuals watching animations of ASL. For instance, researchers in $[12,13]$ found that native ASL signers in an experimental study, with animations displayed at several different fundamental rates, preferred animations with a rate of 0.9 signs per second. Presumably, the slower rate preferred for animation (compared to that for human signers [9]) is due to the animation being more difficult to understand than a video of a fluent human signer. As the quality of ASL animations improves, DHH users may prefer faster animations - to more efficiently consume information. As discussed in our "Research Methods," in the user study we conducted with animations displayed to DHH participants, we used animations with a fundamental rate of 0.8 sign per second, after pauses were inserted.

\section{Base Duration in Animation Systems}

Most sign-language animation systems include a lexicon of individual signs that can be assembled into longer sequences. In such systems, each individual word entry in the lexicon will generally include information about the base duration of the sign (i.e. its default time duration). The source of this timing information has varied: In some cases, designers of individual words stored in the lexicon may have simply created animations without consulting any reference. Whereas, when creating the lexicon for the eSign system [16], the researchers chose the duration of each word by examining the speed of human signers in videos [16]. Other researchers used recordings of human signers more directly: Segouat and Braffort [26] used rotoscopy to create a French Sign Language corpus, with the timing of the motion-data in their corpus based on the timing of the original video recording upon which each was based.

\section{Differential Signing Rate in Animation Systems}

As discussed in the "Goal of Our Research," one of the aims of our study is to create a model that can predict the differential rate of speed of individual words in an ASL animation. In prior work, the rule-based system of AdamoVillani and Wilbur [1] included an automatic rule for one form of differential rate modification: phrase-final lengthening of words. Specifically, they increased the length of the final sign in each phrase, following the prior findings of Wilbur [29]. The 2008 Model [12] included similar phrase-final lengthening, with signs extended $8 \%$ when immediately before a clause boundary and $12 \%$ when immediately before a sentence boundary (these percentages were based on linguistic findings in [9]). In addition, the 2008 Model modified the differential rate of signs (to become faster or slower) depending on whether a particular word had previously appeared in the passage (and whether its subsequent appearance was in the same syntactic position as its prior appearance). As described in our "Research Methods," we seek to create a machine-learning based model, which utilizes a variety of linguistic features.

\section{Pause Insertion in Animation Systems}

Few prior sign-language animation systems have attempted to predict where pauses should be inserted during the signing. Most simply ask the human author to indicate pause locations when using a scripting tool - or they simply insert a pause at every sentence boundary, which had been indicated by the human author. One exception is the trainstation announcement system of Ebling and Glauert [6], in which the authors inserted a hand-coded rule that added a brief pause in-between items in a list, but their rule only applied in this narrow context. The rule-based system of Adamo-Villani and Wilbur [1] automatically inserted pauses at specific locations between and within sentences, based on syntactic structural considerations described by Pfau [24]. Finally, the 2008 Model inserted pauses at 25\% of word-boundaries, based on a prioritization scheme described in [12], which considered the entire syntactic parse tree structure of every sentence in the message. As described in our "Research Methods," we investigate training a machine-learning classification model to determine, for every word boundary in a passage, whether a pause should be inserted at that location.

\section{Pause Duration in Animation Systems}

While human authors creating a sign-language animation have the ability to manually adjust the timing of pauses in some scripting systems, e.g. [30], few automatic algorithms for selecting pause duration in sign-language animations have been proposed. An exception is the 2008 Model [12], which chose the duration of the pauses that it inserted via an algorithm based on the sentences' syntactic structure and on a preference for inserting pauses mid-way between previously placed pauses, following the approach of $[10$, 
11]. However, a limitation of this model is that it required the user to provide a complete syntactic parse tree of every sentence in the message, and the algorithm was based on rules and guidance in prior linguistic literature, which itself was based on researchers' observation of a small number of videos of ASL. We would instead prefer a model of ASL timing based on actual behavioral data and movements of native ASL signers performing fluent ASL passages.

\section{RESEARCH QUESTIONS}

Therefore, we investigate the design and evaluation of machine-learning based models of ASL speed and timing, with a variety of input from DHH participants: (a) We analyze the movements of ASL signers contained within a motion-capture corpus of ASL, for training and evaluating these models, and (b) we conduct a study with DHH participants to understand their impression of the quality of ASL animations resulting from these models. In this way, we investigate the following research questions:

RQ1: In a cross-validation study (where models are trained and tested on various partitions of a dataset), does our model of where human ASL signers insert pauses in their ASL signing have higher accuracy than baseline models (predicting pauses at sentence boundaries only) or the prior 2008 Model $[12,13]$ ?

RQ2: In a cross-validation study, will our model of the time-duration of pauses outperform a baseline (uniform duration) or the 2008 Model $[12,13]$ ?

RQ3: In a cross-validation study, will our model of differential signing rate outperform a baseline (uniform speed) or the prior 2008 Model $[12,13]$ ?

RQ4: Do our new predictive models of pause insertion, pause duration, and individual sign speed lead to animations of ASL that DHH users judge to be understandable in a qualitative feedback study?

\section{RESEARCH METHODS}

Our research methodology consists of four phrases:

Phase 1: We process a pre-existing corpus of video and motion-capture recordings of ASL signers [18], which has been linguistically labeled with words and some phrase boundaries, to prepare a dataset with relevant features for training machinelearning models of our three timing values.

Phase 2: We train and evaluate the accuracy of three models (for differential rate, pause insertion, and pause duration) though five-fold cross-validation of our dataset, and we compare each model to a baseline prediction technique (e.g. predicting pauses at ends of sentences or predicting uniform differential signing rate or pause duration).

Phase 3: We add additional linguistic annotation (complete syntactic parse for each sentence) to a subset of our training corpus so that we can compare the predictions of the 2008 Model $[12,13]$ (which required such data) to the performance of our new model, on these ASL performances.

Phase 4: We conduct a study with DHH participants (who are ASL signers with native fluency) to obtain first-hand feedback and judgements from these users about the quality and naturalness of the animations resulting from our model - along with more general input from these users about what aspects of speed/timing they deem most important when viewing animations of ASL.

Phase 1: Extracting Speed \& Timing from ASL Corpora We make use of the ASL Motion-Capture Corpus [18], which consists of video recordings of native ASL signers performing multi-sentence, unscripted passages in ASL. The corpus contains video and motion-capture data recordings of the handshape, hand movements, body position, and other details, and the data was subsequently annotated by a team of Deaf native ASL signers and linguists, who labeled the individual words, as well as some syntactic phrase boundaries (noun phrases, verb phrases, clauses, sentences). We used the first release of this corpus [18], which consists of 83 passages, performed by a total of 3 ASL signers, containing a total of 7,138 words.

To create a dataset for our research, we needed to process this corpus. Specifically, we calculated a "comma separated values" (CSV) spreadsheet file for each story in the corpus, with the following structure: Each row represented a single word in the corpus. The columns of the file represented key linguistic features (which we intended to use for training our models) or the output predictions for the three timing variables (Did a pause occur after this word? What was the time duration of this pause? What was the differential signing speed of this word?). As shown in Table 1, the set of "predictor features" included information about whether this word was adjacent to a syntactic phrase boundary, the length of the current phrase or sentence in which the word occurs, how far this word is from the nearest pause in the signing, and some numerical measure of how major the syntactic boundary is that immediately follows this word.

The selection of features was inspired by the properties used in prior rule-based methods of predicting speed and timing, e.g. $[12,13]$. All numerical features shown in the table were scaled using unity-based normalization. Table 1 also indicates which of our three models made use of each feature; that topic will be discussed in "Phase 2."

\section{Assumptions Used to Estimate Differential Speed}

As discussed above, there are challenges in making use of ASL recordings to determine differential rate, since the observed timing of a word in a recording is also based on the signer's fundamental rate and the word's base duration. While we can estimate the fundamental rate of the signer across a large sample of recordings, it is more difficult to estimate the base duration of each word. Without having 


\begin{tabular}{|c|c|c|c|c|}
\hline Predictor Features Used in Each Model & $\begin{array}{l}\text { Feature } \\
\text { Type }\end{array}$ & $\begin{array}{c}\text { Pause } \\
\text { Insertion }\end{array}$ & $\begin{array}{l}\text { Differential } \\
\text { Rate }\end{array}$ & $\begin{array}{c}\text { Pause } \\
\text { Duration }\end{array}$ \\
\hline $\begin{array}{l}\text { \#1-4: Is the gap after this word on the boundary of a sentence, } \\
\text { clause, noun phrase, or verb phrase? }\end{array}$ & Yes / No & $\checkmark$ & $\checkmark$ & $\checkmark$ \\
\hline $\begin{array}{l}\text { \#5: Relative Proximity: How close is the gap after this word to } \\
\text { the midpoint between the two nearest pauses? }\end{array}$ & Numerical & $\checkmark$ & $\checkmark$ & $\checkmark$ \\
\hline $\begin{array}{l}\text { \#6: Complexity Index: Value indicating the syntactic importance } \\
\text { of this gap (ranging 1-4) with value of } 4 \text { at sentence boundaries. }{ }^{1}\end{array}$ & Numerical & $\checkmark$ & $\sqrt{ }$ & $\checkmark$ \\
\hline $\begin{array}{l}\text { \#7-9: How many words are in the current sentence, noun phrase, } \\
\text { and verb phrase (if applicable)? }\end{array}$ & Numerical & $\sqrt{ }$ & $\checkmark$ & $\checkmark$ \\
\hline $\begin{array}{l}\text { \#10-11: Is there a pause immediately before or immediately } \\
\text { after this word? (This is output of the "Pause Insertion" model.) }\end{array}$ & Yes / No & $x$ & $\checkmark$ & $\checkmark$ \\
\hline $\begin{array}{l}\text { \#12-13: How far is this word from the beginning of the current } \\
\text { sentence? From the end? }\end{array}$ & Numerical & $x$ & $\sqrt{ }$ & $\checkmark$ \\
\hline $\begin{array}{l}\text { \#14-15: What is the differential rate for the current word and the } \\
\text { following one? (This is the output of the Diff. Rate model.) }\end{array}$ & Numerical & $\mathbf{x}$ & $x$ & $\checkmark$ \\
\hline
\end{tabular}

Table 1: List of Predictor Features Used in this Study, a Checkmarks Indicating if Used in Each of Our Three Models

hundreds of recordings of each word, as performed by a variety of signers, it is difficult to estimate what the "normal" base duration of each word is in ASL. For this reason, some researchers will examine speed or acceleration curves for hand movements during signing, e.g. [5], in lieu of considering the final duration of a word in a recording, when calculating differential rate. For our work, we have estimated the differential rate for each word using the following procedure: We focus on the movement of the signer's dominant hand (right hand of a right-handed person) only, and we omit any time frames during the word when the hand is stationary (since some signs contain periods of time when the hands make contact with the body or remain in place for short period of time). Next, we calculate the average velocity of the hand during these remaining time frames. Finally, we divide by the fundamental signing rate for this signer, as calculated across all recordings of this person in the corpus. In this way, if a word is performed more quickly in some context, we expect a differential rate greater than 1, and vice versa.

\section{Assumptions Used to Estimate Pause Insertion \& Duration} Our corpus did not include human judgements about where pauses were occurring nor what their duration was; thus, we also needed to estimate these values by processing the movement data in our recordings. A challenge is that many ASL signs will contain brief moments of time that are often referred to as "holds," in which the hand is momentarily stationary - and often these occur at the end of a sign. Such brief holds that are part of a sign performance are not generally considered to be a pause (i.e. they are part of the performance of the sign itself lexically, rather than being syntactically motivated prosodic phenomena). Thus, we needed to "filter" out these short holds at the end of words, to identify the slightly longer moments when the hands are stationary that reflect true "pauses." Taking guidance from Grosjean et al. [9] who observed that pauses occur at $25 \%$ of inter-sign locations, we extracted the end-of-sign stationary-hand durations for all words in the corpus, ranked these values, and decided that the top quartile of these values were "pauses." Furthermore, we determined that the "duration" of each of these pauses would be the amount of stationary-hand duration that was in excess of the duration value threshold that defined the top quartile.

\section{Phase 2: Design and Baseline-Evaluation of Models} In this Phase, we implement three machine-learning models to address various aspects of ASL speed and timing. Our models were cascaded, that is, an ordering was established among them such that the output of prior models could be provided as an input feature to a subsequent model:

1. Pause Insertion: The first model was a classification model to determine if a pause should be inserted after the current word. The aforementioned Table 1 lists the various predictor features used in each model; the Pause Insertion model used the first nine features in the table, including: whether the current location was phrase boundary, the syntactic importance of the boundary $^{1}$, and the proximity to nearby pauses ${ }^{2}$.

2. Differential Rate: The second model was a regression model to predict the change in signing rate within the ASL sentence. As shown in Table 1, this model used four more features than the Pause Insertion model (e.g. how close this word was to the end of the sentence). In addition, the Differential Rate model used the output of

\footnotetext{
${ }^{1}$ This property is referred to as the "complexity index" when used in the 2008 Model $[12,13]$ (where a detailed formula is included); we replicate this feature here, with a small modification. While $[12,13]$ depended upon knowing the full syntactic parse tree of the sentence, we do not make this assumption for our model. Thus the "complexity index" used as a feature in our model training is based on the limited syntactic information available in our corpus (boundaries of sentences, clauses, verb phrases, or noun phrases).

2 This property is referred to as "relative proximity" when used in the "2008 Model" [12, 13], with formula appearing in [12].
} 
the Pause Insertion model as one of its input "predictor" features (to consider whether a pause had been inserted before or after the current word, which might suggest it would be performed more slowly, as the signer anticipated or resumed from a pause).

3. Pause Duration: This third model was a regression model to predict the length of each pause. This Pause Duration model logically depends on the results of Pause Insertion, since pause durations need only be calculated where pauses will occur. Furthermore, we utilized the output of the Differential Rate model as one of the input features to this model; specifically, we anticipate that pauses occurring near words with longer duration will themselves be longer in duration.

Design and Cross-Validation of the Pause Insertion Model Since we expected dependencies between whether a pause is inserted at subsequent gap locations (i.e. if a pause is inserted after one word, another is unlikely after the next word), we trained a Linear-Chain Conditional Random Field (CRF) model [4, 31], using [23]. Thus, we treated this task as a sequence-tagging problem (with a $+/-1$ context window, i.e. so that we consider the predictor features of the inter-sign gap immediately preceding and following the current inter-sign gap). We implemented a 5-fold crossvalidation procedure, dividing our data into $80 \%$ training set and $20 \%$ testing set at each evaluation fold. We calculated the average accuracy and f-score across the 5 folds. To select the best working parameters for this model, we performed a grid-search to optimize performance.

Table 2 shows the accuracy and F-score for the model. For comparison, we also present results for a baseline that inserts a pause at the end of every sentence. The rationale for this baseline is that if a human animator were to create an animation and manually chose to insert some pauses, the animator may be likely to put them at all of the sentence boundaries, as a simple approach. The linear-chain CRF model beat this baseline, with an accuracy of $80 \%$ and Fscore slightly exceeding the baseline.

\section{Design and Cross-Validation of Differential Rate Model}

Differential Signing Rate was modeled using regression, to predict a value that represents a multiplier to modify the Base Duration of a sign. We compared performance of Ada Boost, Gradient Boosting, Random Forest, and Extra Trees Regressors [4, 31]; we found Gradient Boosting Regressor [27] to have best performance. We used GridSearchCV to exhaustively search the parameter space. For instance, values of [50 to 400] were explored for the number of estimators (n_estimators), and we found that a value of 50 led to the best result on our training dataset, while avoiding overfitting. Table 3 shows the performance of our model, as compared to a simple baseline (i.e. predicting a uniform signing rate for all signs, specifically a "multiplier" of 1.0). Our model outperformed the baseline; a lower value for "Root Mean Squared Error" (RMSE) error is a better result.

\begin{tabular}{|l|c|}
\hline \multicolumn{1}{|c|}{ Classifier } & Accuracy (F1-Score) \\
\hline Linear-Chain CRF & $\mathbf{0 . 8 0}$ \\
\hline Baseline & 0.77 \\
\hline
\end{tabular}

Table 2: Pause Prediction Model Results

\begin{tabular}{|l|c|}
\hline \multicolumn{1}{|c|}{ Regression Model } & RMSE \\
\hline Gradient Boosting Regressor & $\mathbf{0 . 4 5}$ \\
\hline Baseline & 0.50 \\
\hline
\end{tabular}

Table 3: Differential Signing Rate Prediction Model Results

\begin{tabular}{|l|c|}
\hline \multicolumn{1}{|c|}{ Regression Model } & RMSE \\
\hline Pause Duration (GBR) & $\mathbf{2 . 7 7}$ \\
\hline Baseline & 4.47 \\
\hline
\end{tabular}

Table 4: Pause Duration Prediction Model Results

\section{Design and Cross-Validation of the Pause Duration Model} Pause Duration was also modeled using regression, in this case, to predict a value for the time duration of the pause between two signs. We followed a similar training and testing model-comparison procedure as above, ultimately using a Gradient Boosting Regressor. Our grid search of the parameter space found that n_estimators $=100$ had the best performance on our training dataset. Table 4 shows the performance of the Pause Duration regression model, as compared to a simple baseline (predicting uniform pause duration, specifically the average duration of all pauses in the corpus). Our model outperformed the baseline.

\section{Phase 3: Comparing Our New Model to the 2008 Model}

While Phase 2 showed that our model out-performed a baseline model (inserting pauses at sentence boundaries only, with uniform pause length and uniform sign speed), a better test of this new model would be to compare it against the current state-of-the-art model for speed and pausing in ASL, the 2008 Model of Huenerfauth [12, 13]. Aside from comparing model accuracy, there are other points of comparison between these models:

- The 2008 Model [12, 13] is a rule-based approach based on linguistics studies $[9,10,11]$ that considered a relatively small number of ASL videos. Models based on larger datasets of human signing performance may be more accurate. Furthermore, the 2008 Model requires a full syntactic parse of every sentence as an input for its algorithms; this may be time-consuming for the human author of the ASL message to provide.

- Our new model, which we refer to as "ASL-Speed" is a collection of machine-learning models trained on human behavioral data, from a motion-capture corpus of ASL. ASL-Speed requires features 1-4 (Table 1) as input from an ASL-knowledgeable human user, which uses less input data than required by the Huenerfauth [12] model, which needed a full syntactic parse tree. 


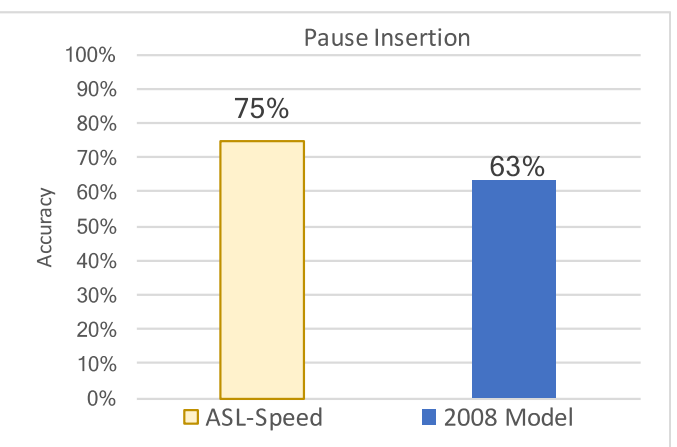

Figure 2: Comparison of our new ASL-Speed model and the 2008 Model on the Pause Insertion task - for a subset of passages from [17] for which we added syntax annotation.

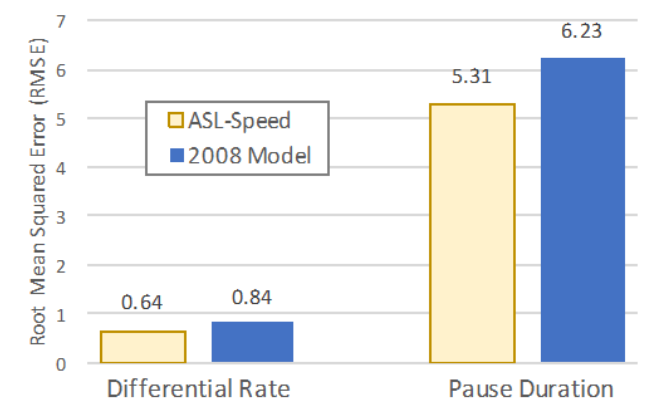

Figure 3: Comparison among new ASL-Speed model and the 2008 Model - for Differential Rate and Pause Duration.

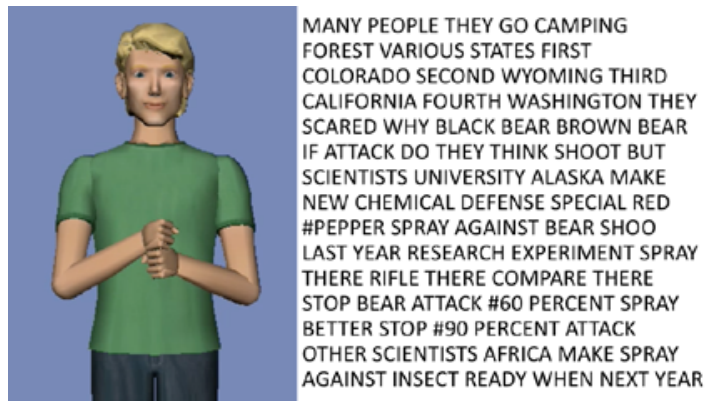

Figure 4: Image of animation (left) seen participants in Phase 4 , and transcript (right). Participants did not see transcript.

Unfortunately, given the need for a full syntactic parse of all sentences in order to run the 2008 Model, running a test on the entire corpus [18] was impractical, since the original annotation of that corpus did not include such annotation. The corpus contained 83 multi-sentence passages, from a total of three signers. For this comparative analysis between ASL-Speed and the 2008 Model, we had to use a subset of the corpus. We selected three passages from each signer (selecting the three with length closest to the median for each person); there were 958 words total in the 9 passages. Next, an ASL linguist annotated each passage with a full syntactic parse tree. On this small testing set, we ran our new ASL-Speed model and the 2008 Model [12]. These passages were excluded from our models' training set.
Figure 2 shows the prediction accuracy for the new ASLSpeed model and 2008 Model for the Pause-Insertion task. The new ASL-Speed model had higher accuracy than the 2008 Model. Given that the 2008 Model had been the previous state-of-the-art method of predicting this information for ASL animations, this is an important indicator of the quality of our new model.

Figure 3 shows the prediction accuracy for the two regression tasks: Differential Rate and Pause Duration Lower values for Root Mean Squared Error (RMSE) indicates better performance. The new ASL-Speed models outperformed the 2008 Model. Once again, this is an important result, since the 2008 Model had previously been the state-of-the-art method for predicting these speed and timing values for ASL animation.

\section{Phase 4: User-Study with Subjective Feedback}

While this metric-based analysis above is useful, it is important to test animations produced from these models with participants who are native ASL signers, to understand their reaction to these animations - and to ask for additional recommendations or feedback about how to improve them, as in [15]. For this evaluation, we chose an interview-based study design, in which a native ASL signer who was a researcher on our team met with Deaf native ASL signers to discuss their views on speed and timing in ASL animations, while looking at animations from our new model.

\section{Procedure and Data Collection}

A total of $8 \mathrm{DHH}$ participants were recruited on the Rochester Institute of Technology campus. Participants included 2 men and 6 women, of ages 21 to 34 (median age 23). Participants were native ASL signers: All participants learned ASL at their childhood (before age 3), 5 having Deaf parents, all of them having used ASL at school as a young child, and 5 with other Deaf family members. The researcher (also a Deaf native ASL signer) met the participants to conduct the interview in ASL.

After some demographic questions, participants were asked to look at a laptop that displayed three pairs of animations of ASL (Figure 4). Each pair (shown side-by-side) was an identical passage, but one animation used speed and timing based on our new ASL-Speed model, while the other was based on the baseline models described previously, i.e. pauses at end of sentences and uniform sign speed and pause duration. The passages were selected from the stimuli shown in $[12,13]$, and each passage was approximately 75 words in length, on various topics (scientists developing a bear repellent spray, increases in rice prices, and a student selecting a career). Animations were generated using Sign Smith Studio [30], which allows for control of time parameters during animations, as described in $[12,13]$.

The semi-structured interview focused on participants' impressions of the animations' quality, which animation they preferred in each pair, and whether they noticed a difference between the two. When displaying the first pair 
of animations, we did not inform participants that speed or pauses differed; we wanted to see if participants noticed this on their own. Most of the participants (6 of 8) preferred the ASL-Speed animations, compared to the baseline one.

\section{Some comments were about the overall speed, in general:}

- "The [baseline] video was slow. If the animation signs slow, it means itself is a beginner or rookie. The [new] was fast. It means it's an experienced signer." (P2) ${ }^{3}$

- "The [baseline] is good and has a good pace. The [new] is clearer than the [baseline]. It has strong ASL content than the [baseline]. It is clear, but fast." (P3)

- "The [new] has a good pace. [Baseline] is fast." (P6)

Then, we asked them some questions in regard to speed and timing, e.g. whether the boundaries between sentences were easy to perceive in the animations, whether the pauses in each animation seemed natural, whether the overall speed and timing of the animations seemed natural. Throughout the course of the interview, participants had an opportunity to view all three pairs of ASL animations, while commenting on these topics. The sequence of displaying the animations was rotated across participants, as well as whether the ASL-Speed animation was on the left or right.

\section{Some participants commented on the pausing:}

- "[New] is fine with the pausing. It needs to improve quality. I suggest add a few pauses, but sign consistently." (P2)

- "[New] is okay... Need to add 2 pauses. If no pause, I would be overwhelmed. That wouldn't be clear." (P3)

- "I notice there are pauses [in the new]. The flow of the content is good and smooth." (P4)

- "[Baseline] needs to add pauses between sentences, not continuing sign too fast. I lose information if it signs too fast." (P4)

One participant preferred the baseline animation in one pair, but he preferred the ASL-Speed in other pairs. In regard to the first pair, P6 commented "It [baseline] has no pauses. It [new] pauses at the wrong time." For the pairs of animations for which the ASL-Speed was preferred, P6 said "It [new] is normal, almost like a real person signing. It's better than the first one [baseline]. " and "It's good [new]"

Finally, some comments were about the signing speed:

- “[Baseline] seems for beginners, and [new], for experience signers... [New] is not too fast; it is regular speed like a conversation... [New] is close enough to match the natural speed of an ASL signer." (P02)

- " "[New] is fine and steady." (P3)

- "[New] is the right speed" (P5)

\footnotetext{
${ }^{3}$ Participants did not know which animation was based on the baseline model or on the new model; they referred to the videos by pointing to them. We have edited their comments to indicate "[baseline]" or “[new]" for each participant (e.g. P2, P3, etc.). The comments were transcribed into English by the Deaf researcher.
}

Individual participants may differ in the speed they prefer to receive animated signing, e.g. due to language-fluency, demographic, or experience factors, as discussed in [17].

In summary, 2 participants preferred a baseline animation commenting that they preferred animation (they perceived as) having no pauses to an animation with a pause in a location they judged to be incorrect. This insight suggests that we should consider the precision/recall tradeoff of our pause insertion model. The other 6 participants preferred ASL-Speed animations - commenting positively about speed/pausing. While this was a relatively small interview study, the comments indicated a sensitivity to speed and pausing in these animations (as had been found in [13]), with a preference for the new model over the baseline. As discussed previously, this baseline (insert pauses at end of sentences only, use uniform signing rate and uniform duration for pauses) is how nearly all current sign-language animation systems generate their speed and pausing values.

\section{Discussion: Deaf Community Concerns}

Conducting studies with DHH participants who are asked to evaluate animations of ASL is essential for ensuring that the resulting technology is actually understandable and useful for this community. This concern is underscored by recent announcements by major international organizations representing segments of the Deaf community. Given the importance of these organizations within the community and the direct relevance of these statements to ASL animation technology, we believe it is important to discuss these concerns, before proceeding to summarize the conclusions from this research study below.

On March 14, 2018, the World Federation of the Deaf (WFD) and the World Association of Sign Language Interpreters (WASLI) issued a joint statement expressing concern "with the way in which decisions on where and when to use signing avatars as a form of access to spoken or written content is being managed by public authorities” [32]. The statement reminds readers that sign languages are full languages, and that word-for-word transliterations are rarely possible; thus, human judgement is necessary to address context and cultural aspects in translation, e.g. "Whilst the technology has progressed and offers real potential for wider use of signing avatars, these computerized products do not surpass the natural quality and skill provided by appropriately trained and qualified interpreters and translators” [32].

The statement especially cautions against use in particular contexts, i.e. "when the information being delivered is live, complex or of significant importance to the lives of deaf citizens such as news broadcasts, public emergency announcements, or political announcements.” However, the statement does acknowledge that this technology "might be used for pre-recorded static customer information, for example, in hotels or train stations where instructions might be given about where to check in or queue up. This is acceptable as long as deaf people have been involved in 
advising on the appropriateness of the signed sentences, and that there is no interaction or 'live' signing required."

\section{Ethical Implications for Researchers in this Field}

As researchers working on this technology, we speculate that this statement may be, in part, in response to proliferation of online news articles or videos about research or prototype sign-language technologies - which may not accurately convey the present limitations of such systems when communicating to a popular audience on social media. We speculate that this statement may also be in response to media or advertising coverage of commercial efforts, some of which may suggest that technologies available now (or in the near future) will have performance that is actually beyond the state of the art of sign language animation, understanding, or translation technologies.

A danger from such inaccurate dissemination is that decision-makers within companies, educational institutions, or governments may believe that current accessibility services (e.g. professional interpreters) could be replaced by these technologies, as a cost-savings measure. Prior research has examined legal aspects of this issue, in regard to compliance with guidelines [21], and there has been other evidence that members of the Deaf community are concerned that automated accessibility services will have lower quality than professional services they currently receive, such as sign language interpreting, especially in critical environments like education or healthcare. Such concerns have emerged in surveys of DHH users [25], as discussed in [3]. Thus, researchers in this field have a responsibility to measure the usability of systems before deployment (and to carefully communicate the potential and the limitations of their work to a broader audience).

As discussed at the beginning of this paper, our research goal is not to supplant high-quality accessibility services provided by human interpreters, but rather we seek to increase the prevalence of sign language content on websites. Notably, we are not proposing immediate commercial deployment, but rather we disseminate our work through academic venues, and the applications we discuss for our technologies do not assume technology that is well beyond the state of the art, i.e. automatic machine translation of English to ASL with high accuracy for a wide variety of texts. Instead, we are proposing a technology with a human-in-the-loop (in particular, it may be a Deaf individual who is a fluent ASL signer who authors the script for generating animations in the application we envision for our technology). Given that we are designing technologies for the Deaf community, our laboratory has also conducted this work in a context where multiple DHH individuals are part of our team, and we are basing the movements and other details of our virtual signer on recordings of ASL signers, as linguistically labeled by Deaf ASL signers and linguists. Finally, it is important to carefully evaluate the quality of the resulting technology: both in the form of computational linguistic evaluating using cross-validation method of the intrinsic model quality, but also by obtaining feedback from people who are Deaf ASL signers about the resulting animations.

\section{CONCLUSIONS, LIMITATIONS, AND FUTURE WORK}

In this work, we have characterized the speed and timing approaches employed in prior sign-language animation systems, and we have presented a new method, based on machine-learning models trained on a motion-capture corpus of actual ASL signers, for automatically calculating three key values: selection of locations to insert pauses, setting the differential speed of individual words, and adjusting the time duration of the pauses. Since the model was trained on a small corpus, we promoted generalizability by normalizing the fundamental speed of each human recording prior to training, and designed the model such that no features were lexically specific (to avoid out-ofvocabulary issues). Our model has out-performed a baseline method of setting these values (which is the commonly used approach in most prior sign-language animation systems) of pausing only at the end of sentences and using uniform sign speed and pause duration. In addition, we have compared our model to the prior state-of-the-art approach [12, 13], and we found that our system outperformed this prior model in predicting timing values for a set of ASL passages. As a final evaluation, we conducted a small study with interviews of Deaf native ASL signers who examined animations based on our models, to determine whether they prefer these to animations driven by a baseline model. We found that participants preferred our animations, and they offered comments about the importance of speed and timing in ASL animations. Finally, we have commented on issues regarding the responsibilities of researchers working in this area, in light of recent statements from major international Deaf organizations.

One limitation of our current approach is that we treat the differential rate modifications of ASL signs as a multiplier; the actual speed adjustments may, in fact, consist of acceleration profiles which affect particular sub-portions of each sign, and we intend to explore such possibilities in future work. In addition, our comparison to the prior 2008 Model was based on only a subset of the full corpus of [18] for which we labeled some syntactic information; we plan to annotate additional data and repeat this analysis with a larger dataset in future work. After examining these additional modeling improvements and cross-validation evaluations, we plan to conduct a larger experimental study with Deaf native ASL signers, to evaluate the animations. Our goal is to create technology to facilitate the authoring of sign-language animations by automating their synthesis, to promote additional sign-language content on the web.

\section{ACKNOWLEGMENTS}

This material is based upon work supported by the National Science Foundation under awards 1400802, 1462280, and 1746056. We are grateful for the contribution of Abraham Glasser in data collection from participants for this work. 


\section{REFERENCES}

[1] Nicoletta Adamo-Villani and Ronnie Wilbur. 2015. ASL-Pro: American Sign Language Animation with Prosodic Elements. In: Antona M., Stephanidis C. (eds) Universal Access in Human-Computer Interaction. Access to Interaction. UAHCI 2015. Lecture Notes in Computer Science, vol 9176. Springer, Cham. https://doi.org/10.1007/978-3-31920681-3_29

[2] Ursula Bellugi and Fischer Susan. 1972. A Comparison of Sign Language and Spoken Language. Cognition 1, 173-200. https://doi.org/10.1016/0010-0277(72)90018-2

[3] Larwan Berke, Sushant Kafle, and Matt Huenerfauth. 2018. Methods for Evaluation of Imperfect Captioning Tools by Deaf or Hard-of-Hearing Users at Different Reading Literacy Levels. In Proceedings of the ACM CHI Conference on Human Factors in Computing Systems (CHI'18). ACM, New York, NY, USA. https://doi.org/10.1145/3173574.3173665

[4] Christopher Bishop. 2011. Pattern Recognition and Machine Learning (Information Science and Statistics). Springer, New York, New York, USA.

[5] Kyle Duarte. 2012. Motion capture and avatars as portals for analyzing the linguistic structure of signed languages. Doctoral Dissertation, Université de Bretagne Sud.

[6] Sarah Ebling and John Glauert. 2016. Building a Swiss German Sign Language avatar with JASigning and evaluating it among the Deaf community. Univers. Access Inf. Soc. 15, 4 (Nov 2016), 577-587. http://dx.doi.org/10.1007/s10209-015-0408-1

[7] Susan Fischer, Lorraine Delhorne, and Charlotte Reed. 1999. Effects of Rate of Presentation on the Reception of American Sign Language. Journal of Speech, Language, and Hearing Research 42, 3, 568-582. https://doi.org/10.1044/jslhr.4203.568

[8] François Grosjean. 1977. The Perception of Rate in Spoken and Sign Languages. Perception \& Psychophysics 22, 4, 408-413. https://doi.org/10.3758/BF03199708

[9] François Grosjean. 1979. A Study of Timing in a Manual and a Spoken Language: American Sign Language and English. Journal of Psycholinguistic Research 8, 4, 379-405. http://dx.doi.org/10.1007/BF01067141

[10] Francois Grosjean, Lysiane Grosjean, and Harlan Lane. 1979. The Patterns of Silence: Performance Structures in Sentence Production. Cognitive Psychology 11, 58-81. https://doi.org/10.1016/00100285(79)90004-5
[11] Francois Grosjean and Harlan Lane. 1977. Pauses and Syntax in American Sign Language. Cognition 5, 2, 101-117. https://doi.org/10.1016/00100277(77)90006-3

[12] Matt Huenerfauth. 2009. A Linguistically Motivated Model for Speed and Pausing in Animations of American Sign Language. ACM Trans. Access. Comput. 2, 2, Article 9 (June 2009), 31 pages. http://dx.doi.org/10.1145/1530064.1530067

[13] Matt Huenerfauth. 2008. Evaluation of a psycholinguistically motivated timing model for animations of American Sign Language. In Proceedings of the 10th international ACM SIGACCESS conference on Computers and accessibility (Assets '08). ACM, NY, NY, USA, 129136. http://dx.doi.org/10.1145/1414471.1414496

[14] Matt Huenerfauth. 2014. Learning to generate understandable animations of American Sign Language. In Proceedings of the 2nd Annual Effective Access Technologies Conference, Rochester Institute of Technology, Rochester, NY, USA. 6 pp. http://scholarworks.rit.edu/eatc/Papers/Papers/4/

[15] Matt Huenerfauth, Liming Zhao, Erdan Gu, and Jan Allbeck. 2007. Evaluating American Sign Language generation through the participation of native ASL signers. In Proceedings of the 9th international ACM SIGACCESS conference on Computers and accessibility (Assets '07). ACM, NY, NY, USA, 211218. https://doi.org/10.1145/1296843.1296879

[16] Vince Jennings, Ralph Elliott, Richard Kennaway, and John Glauert. 2010. Requirements for a signing avatar. in Proceedings of the Workshop on Corpora and Sign Language Technologies (CSLT), LREC'10.

[17] Hernisa Kacorri, Matt Huenerfauth, Sarah Ebling, Kasmira Patel, Kellie Menzies, and Mackenzie Willard. 2017. Regression Analysis of Demographic and Technology-Experience Factors Influencing Acceptance of Sign Language Animation. ACM Trans. Access. Comput. 10, 1, Article 3 (April 2017), 33 pages. DOI: https://doi.org/10.1145/3046787

[18] Pengfei Lu and Matt Huenerfauth. Collecting and evaluating the CUNY ASL corpus for research on American Sign Language animation. 2014. Computer Speech \& Language 28, 3 (May 2014), 812-831. http://dx.doi.org/10.1016/j.csl.2013.10.004

[19] Ross Mitchell, Travas Young, Bellamie Bachleda, and Michael Karchmer. 2006. How Many People Use ASL in the United States? Why Estimates Need Updating. Sign Language Studies 6, 3 (Jan 2006). https://doi.org/10.1093/deafed/enj004

[20] Carol Neidle and Christian Vogler. 2012. A New Web Interface to Facilitate Access to Corpora: 
Development of the ASLLRP Data Access Interface, In Proceedings of the 5th Workshop on the Representation and Processing of Sign Languages: Interactions between Corpus and Lexicon, LREC 2012, Istanbul, Turkey.

[21] Mark Neerincx, Anita Cremers, Judith Kessens, David van Leeuwen, Khiet Truong. 2009. Attuning speech-enabled interfaces to user and context for inclusive design: technology, methodology and practice. Univers, Access Info. Soc. 8, 2 (2009), 109122. http://dx.doi.org/10.1007/s10209-008-0136-x

[22] Afra Pascual, Mireia Ribera, and Toni Granollers. 2014. Impact of web accessibility barriers on users with hearing impairment. In Proceedings of the XV International Conference on Human Computer Interaction (Interacción '14). ACM, New York, NY, USA, Article 8, 2 pages.

https://doi.org/10.1145/2662253.2662261

[23] Fabian Pedregosa, Gaël Varoquaux, Alexandre Gramfort, Vincent Michel, Bertrand Thirion, Olivier Grisel, Mathieu Blondel, Peter Prettenhofer, Ron Weiss, Vincent Dubourg, Jake Vanderplas, Alexandre Passos, David Cournapeau, Matthieu Brucher, Matthieu Perrot, and Édouard Duchesnay. 2011. Scikit-learn: Machine Learning in Python. J. Mach. Learn. Res. 12 (November 2011), 2825-2830.

[24] Roland Pfau, Markus Steinbach, and Bencie Woll. (eds.). 2012. Sign Language: An International Handbook. 37. Walter de Gruyter.

[25] Soraia Silva Prietch, Napoliana Silva de Souza, and Lucia Villela Leite Filgueiras. 2015. Application requirements for deaf students to use in inclusive classrooms. In Proc. of the Latin American Conf. on Human Computer Interaction (CLIHC '15). ACM, NY, NY, USA, Article 5, 8 pages. http://dx.doi.org/10.1145/2824893.2824898

[26] Jérémie Segouat and Annelies Braffort. 2009. Toward the study of sign language coarticulation: methodology proposal. In Proceedings of the Second International Conferences on Advances in Computer-Human Interactions, Cancun, 2009. 369374. https://doi.org/10.1109/ACHI.2009.25

[27] sklearn.ensemble.GradientBoostingRegressor. 2018. Retrieved April 09, 2018, from http://scikitlearn.org/stable/modules/generated/sklearn.ensemble .GradientBoostingRegressor.html

[28] Carol Bloomquist Traxler. 2000. The Stanford Achievement Test, Ninth Edition: National norming and performance standards for deaf and hard-ofhearing students. Journal of Deaf Studies and Deaf Education 5, 4, 337-348. http://dx.doi.org/10.1093/deafed/5.4.337
[29] Ronnie Wilbur. 2009. Effects of varying rate of signing on ASL manual signs and nonmanual markers. Language and Speech 52(2-3), 245-285. https://dx.doi.org/10.1177\%2F0023830909103174

[30] Vcom3D, Sign Smith Studio. Retrieved April 9, 2018 from http://www.vcom3d.com/signsmith.php

[31] Ian H. Witten and Eibe Frank and Mark A. Hall and Christopher J. Pal. 2016. Data Mining, Fourth Edition: Practical Machine Learning Tools and Techniques. Morgan Kaufmann, Burlington, Massachusetts, USA.

[32] World Federation of the Deaf. 2018. WFD and WASLI Issue Statement on Signing Avatars. Retrieved from https://wfdeaf.org/news/wfd-wasliissue-statement-signing-avatars/ on March 20, 2018. 